\title{
Le métabolisme cellulaire in vitro : effets métaboliques méconnus
}

Les études métaboliques effectuées sur des segments frâ̂chement isolés de tubules rénaux permettent de préciser de nombreux aspects de la fonction métabolique de l'organe. Les conditions particulières que rencontrent cependant les cellules rénales incubées in vitro créent des modifications importantes qu'il faut savoir analyser pour éviter d'aboutir à des conclusions erronées.

\section{Patrick Vinay}

Josette Noël

\section{Alberto Tejedor André Gougoux}

\section{ADRESSES \\ $\mathrm{P}$. Vinay : docteur en médecine, docteur ès scien- ces. J. Noël : master of sciences. A. Tejedor : docteur en médecine, docteur ès sciences. A. Gou- goux : docteur en médecine. Groupe de recher- che en transport membranaire, départe- ments de physiologie et de médecine, uni- versité de Montréal et hôpital Notre-Dame de Montréal, faculté de médecine, CP 7128 succ. A, Montréal (Québec), H3C 3J7, Canada.}

TIRÉS A PART

P. Vinay.

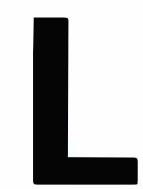

'étude du métabolisme intermédiaire survenant dans des organes spécifiques est un prérequis à la compréhension du métabolisme intermédiaire de l'organisme entier. En effet celui-ci reflète une somme d'événements complémentaires ou compétitifs se produisant à l'échelle cellulaire. Afin de mieux définir la contribution métabolique de chaque organe, un raffinement progressif des méthodes s'est imposé.

L'étude des modifications du sang entrant ou sortant d'un organe s'est avérée être rapidement limitée par l'aspect quantitatif défavorable inhérent à la méthode: une activité métabolique intense ne détermine souvent qu'une faible différence artério-veineuse en raison du flux de perfusion important [1]. De plus, la recirculation des traceurs utilisés, et leurs transformations en dehors de l'organe étudié, introduisent des biais parfois insurmontables.
L'utilisation d'organes isolés et perfusés a amélioré ce problème, la recirculation du milieu d'incubation permettant d'amplifier les signes fonctionnels [2]. Malheureusement cette technique se heurte encore à des difficultés : elle ne permet pas de se libérer de la masse compacte de l'organe, ce qui introduit des incertitudes quant à l'homogénéité de la perfusion. De plus elle ne permet pas de disséquer le rôle spécifique de chaque type cellulaire : cela est d'autant plus ennuyeux qu'une hétérogénéité importante règne même dans des organes apparemment simples comme le foie [3]. Plusieurs populations cellulaires et hépatocytaires s'y retrouvent mêlées, imbriquant leurs caractéristiques métaboliques distinctes et souvent exclusives.

L'utilisation de cellules fraîchement isolées [4] a certainement révolutionné l'approche expérimentale du métabolisme intermédiaire. Non seulement cette technique permet-elle de 
maintenir un échange optimal de chaque cellule avec le milieu d'incubation, mais elle permet aussi la comparaison de multiples conditions expérimentales imposées simultanément à un matériel hautement homogène : sa grande souplesse facilite les études expérimentales nécessitant un protocole complexe [5]. Enfin ce système permet souvent de contourner les difficultés posées par l'hétérogénéité cellulaire sans recourir à la culture de cellules. Celle-ci est très utile, mais s'accompagne souvent de transformations métaboliques profondes.

Différentes techniques, reposant sur la destruction sélective de certains types cellulaires, sur la dissection de régions tissulaires cellulairement homogènes, sur la ségrégation de cellules ou de tubules en fonction de leur densité (Percoll, Ficoll, sucrose), ou sur des marquages cellulaires spécifiques avec séparation en cytométrie de flux, ont été introduites. Ces techniques débutent toutes par l'obtention de cellules (ou de groupes de cellules) fraîchement isolées au moyen d'une digestion enzymatique ou par dissociation mécanique des complexes de jonction et des ponts intercellulaires. Quoique moins précises que les techniques de microdissection pour aborder les problèmes d'hétérogénéité métabolique, ces techniques permettent d'obtenir d'abondantes quantités de tissus relativement purs, et donc d'utiliser avec profit les techniques de mesures métaboliques conventionnelles.

Ces concepts ont été appliqués avec succès au rein où ils ont permis l'étude du métabolisme de différents segments tubulaires isolés [6, 7]. Mais chaque technique a ses problèmes ou ses limites. Nous discuterons ici de quelques-unes des particularités métaboliques introduites par cette technique d'étude lorsqu'elle est appliquée au rein.

\section{Segments tubulaires isolés}

La digestion à la collagénase de tranches tissulaires fines obtenues à partir de différentes régions d'un même rein, suivie ou non d'une étape de purification sur gradient de Percoll, permet d'obtenir une suspension pure de glomérules et au moins trois frac$\mathrm{m} / \mathrm{s} n^{\circ} 3 \mathrm{vol}$. 6 , mars 90

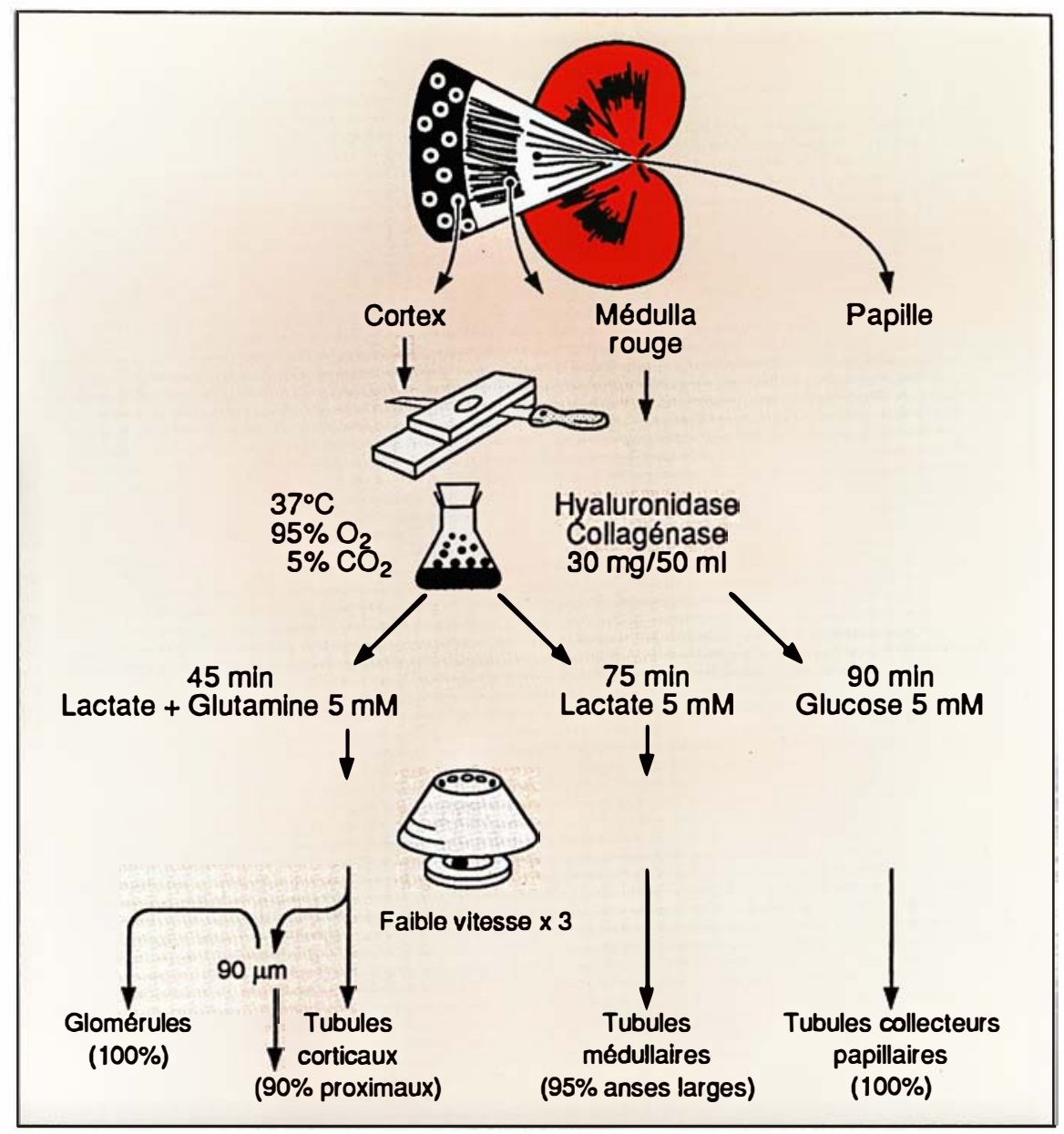

Figure 1. Isolement des segments tubulaires de néphron. Le cortex superficiel, la médulla rouge et la papille du rein sont séparés et tranchés au moyen d'un microtome de Stadie Riggs. Les tranches sont digérées enzymatiquement durant 45-90 minutes en présence de substrats. Les glomérules sont extraits par tamisage et les tubules proximaux, par centrifugation sur gradient de Percoll du produit de digestion cortical. Les anses larges et les collecteurs papillaires sont directement obtenus, respectivement de la médulla rouge et de la papille.

tions distinctes de néphron : tubules proximaux, tubules d'anses larges médullaires et tubules collecteurs papillaires (figure 1). Ces tissus présentent des caractéristiques morphologiques, enzymatiques et métaboliques propres $[6,7]$ : bordures en brosse du tubule proximal (indiquées par la présence de gammaglutamyltranspeptidase $(\gamma-G T)$ et de phosphatase alcaline (PA)), néoglucogenèse exclusivement proximale (présence de phosphoénolpyruvate carboxykinase), glycolyse prédominante dans les anses larges (hexokinase), osmolytes spécifiques dans les tubules collecteurs [8] (Tableau I, p. 270). De courts segments tubulaires sont obtenus, contrairement aux cellules isolées, maintenant ainsi la polarité épithéliale. La lumière de ces segments tubulaires est maintenue ouverte et perméable, tel qu'en témoigne l'accès rapide des substrats solubles aux enzymes de la bordure en brosse [6]. Ces suspensions sont fonctionnellement stables, présentent des récepteurs membranaires intacts $[6,7]$ et sont le siège d'un métabolisme actif (Tableau I). Elles 
Tableau 1

\section{CARACTÉRISTIQUES ENZYMATIQUES ET FONCTIONNELLES DE SEGMENTS PURIFIÉS DE NÉPHRONS DE CHIEN}

\begin{tabular}{|c|c|c|c|}
\hline $\begin{array}{l}\text { Activités enzymatiques } \\
\left(\mu \mathrm{mol} . \mathrm{mg}^{-1} \cdot \mathrm{h}^{-1}\right)\end{array}$ & $\begin{array}{l}\text { Tubules } \\
\text { proximaux }\end{array}$ & $\begin{array}{l}\text { Anses } \\
\text { larges }\end{array}$ & $\begin{array}{l}\text { Tubules } \\
\text { collecteurs }\end{array}$ \\
\hline $\begin{array}{l}\gamma \text {-glutamyltranspeptidase } \\
\text { Phosphatase alcaline } \\
\text { Hexokinase } \\
\text { Phosphoénolpyruvate } \\
\text { Carboxykinase }\end{array}$ & $\begin{aligned} 54 & \pm 3(3) \\
4 & \pm 0,6(5) \\
2 & \pm 0,5(4) \\
33 & \pm 1,9(6)\end{aligned}$ & $\begin{aligned} 6 & \pm 1,3(5) \\
0,5 & \pm 0,1(5) \\
19 & \pm 2,8(4) \\
& N D\end{aligned}$ & $\begin{aligned} 1 & \pm 0,3(4) \\
0,1 & \pm 0,01(8) \\
2 & \pm 1(3) \\
& N D\end{aligned}$ \\
\hline $\begin{array}{l}\text { Activité ATPasique } \\
\left(\mu \mathrm{mol} . \mathrm{mg}^{-1} \cdot \mathrm{h}^{-1}\right)\end{array}$ & $\begin{array}{l}\text { Cortex } \\
(n=12)\end{array}$ & $\begin{array}{l}\text { Médulla rouge } \\
(n=20)\end{array}$ & $\begin{array}{l}\text { Papille } \\
(n=5)\end{array}$ \\
\hline $\begin{array}{l}\text { ATPases totales } \\
\mathrm{Na}^{+}, \mathrm{K}+- \text { ATPase } \\
(\%) \\
\mathrm{H}^{+}-\mathrm{ATPase} \\
(\%)\end{array}$ & $\begin{array}{c}22,7 \pm 2,8 \\
10,9 \pm 1,4 \\
49 \% \\
3,1 \pm 0,4 \\
13 \%\end{array}$ & $\begin{array}{c}88,5 \pm 7,8 \\
70,1 \pm 5,8 \\
79 \% \\
1,0 \pm 0,3 \\
1 \%\end{array}$ & $\begin{array}{c}11,1 \pm 2,3 \\
5,5 \pm 1,1 \\
50 \% \\
0,8 \pm 0,3 \\
7,2 \%\end{array}$ \\
\hline $\begin{array}{l}\text { Métabolisme aérobie } \\
\left(\mu \text { moles.g } g^{-1} \cdot h^{-1}\right)\end{array}$ & 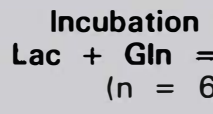 & $\mathrm{mM}$ & $\begin{array}{l}\text { ubation avec } \\
\text { ucose } 2 \mathrm{mM} \\
(\mathrm{n}=25)\end{array}$ \\
\hline $\begin{array}{l}\text { Glucose } \\
\text { Lactate } \\
\text { Glutamine }\end{array}$ & $\begin{array}{r}26 \pm 1,5 \\
-107 \pm 7,4 \\
-\quad 58 \pm 5,2\end{array}$ & $\begin{array}{r}-1 \pm 0,5 \\
-61 \pm 5,7 \\
-18 \pm 1,8\end{array}$ & $\begin{array}{r}-106 \pm 9,5 \\
232 \pm 12,5 \\
-\end{array}$ \\
\hline
\end{tabular}

Les activités enzymatiques sont mesurées par spectrophotométrie sur des homogénats en absence de calcium. L'activité ATPasique totale inclut l'activité de l'ATPase mitochondriale (ATP synthétase). Métabolisme : les valeurs négatives expriment une extraction de substrats et les valeurs positives, une production de métabolites. Moyennes \pm ESM (n). ND = non détectable.

permettent donc d'examiner les relations entre le travail cellulaire et les conditions énergétiques de chacun de ces segments tubulaires [6,9].

\section{Énergétique et respiration tubulaire} Regulation of hepatic metabolism. New York Academic Press 1974: 726-50.

6. Tejedor A, Noël J, Vinay P, Boulanger Y, Gougoux A. Characterization and metabolism of canine proximal tubules, thick ascending limbs, and collecting ducts in suspension. Can J Physiol Pharmacol 1988 ; 66 : 997-1009.

7. Vinay $P$, Gougoux A, Lemieux G. Isolation of a pure suspension of rat proximal tubules. Am J Physiol 1981; 241 : F 403-11.

8. Boulanger $Y$, Legault $P$, Tejedor $A$, Vinay $\mathrm{P}$, Thériault Y. Biochemical characterization and osmolytes in papillary collecting ducts from pig and dog kidneys. Can

Hydrolyse d'ATP. Comme dans toutes les cellules de l'organisme, l'hydrolyse de l'ATP fournit l'énergie requise pour le travail cellulaire. Ainsi la recherche d'ATPases de transport dans les segments de néphron fournit une indication sur leur travail. Celle-ci démontre la présence de deux ATPases majeures dans le néphron proximal : la $\mathrm{Na}^{+}, \mathrm{K}^{+}$-ATPase responsable du transport sodé, localisée sur la membrane basolatérale, et une $\mathrm{H}^{+}$-ATPase, responsable de la sécré- tion de $\mathrm{H}^{+}$, localisée sur la bordure en brosse (Tableau I et figure 2). Au contraire, la $\mathrm{Na}^{+}, \mathrm{K}^{+}$-ATPase prédomine largement dans l'anse large. Les deux types d'ATPases sont retrouvés dans les tubules collecteurs, mais avec un niveau d'activité beaucoup moindre. Des $\mathrm{H}^{+}$-ATPases sont cependant retrouvées sur les deux membranes (apicale et basolatérale) de ce segment. Synthèse d'ATP. Le capital en ATP étant petit par rapport à l'utilisation d'ATP (Tableau II), une synthèse continue et rigoureusement proportionnelle à son utilisation est requise. Celle-ci implique l'internalisation dans la mitochondrie des produits de dégradation de l'ATP, l'ADP et le $\mathrm{Pi}$, où leur liaison est catalysée par l'ATP synthétase selon une réaction couplée à une entrée de protons dans 


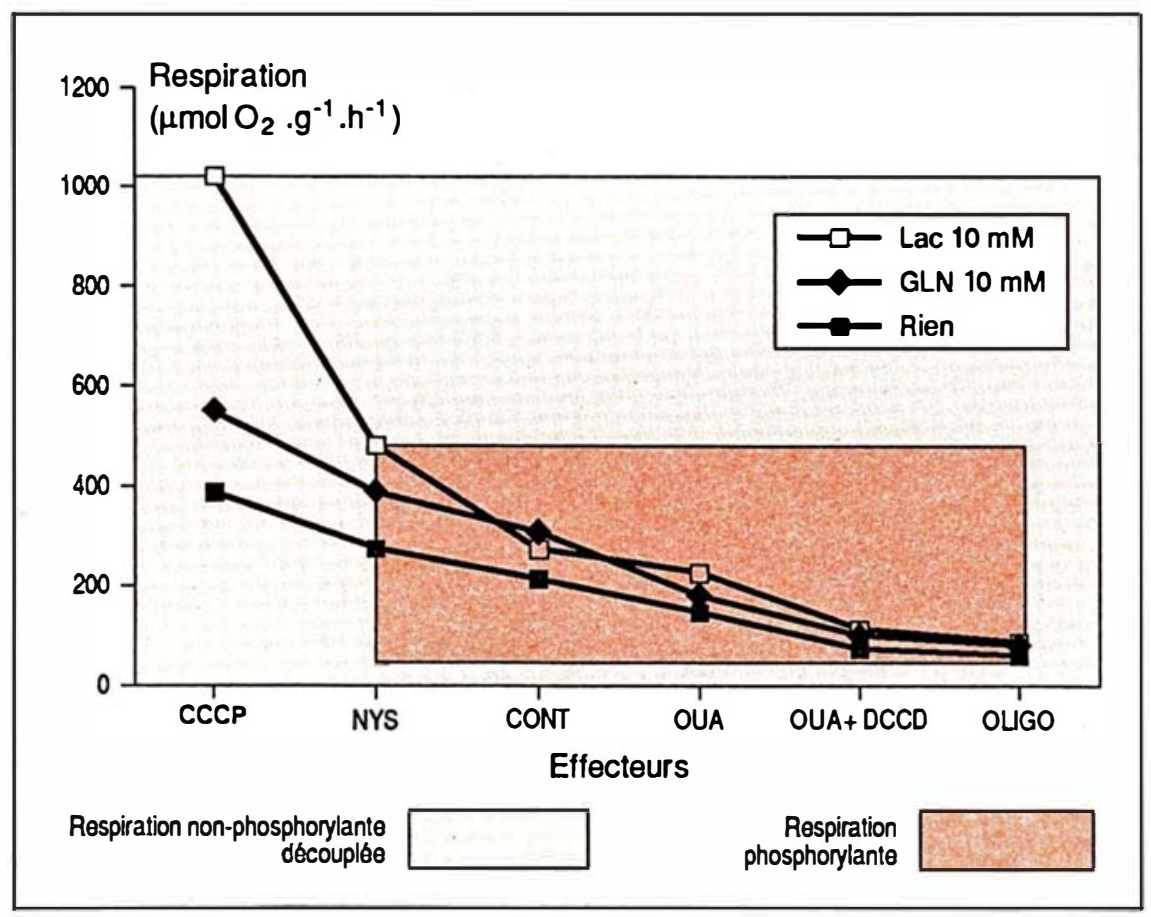

Figure 2. Fourchette maximale de respiration observée sur des tubules proximaux de reins de chien (rectangle clair). La respiration phosphorylante (rectangle foncé) évolue entre O (OLIGO) et $100 \%$ (NYS). Les tubules sont étudiés sous trois conditions de substrats (rien, lactate $10 \mathrm{mM}$, glutamine $10 \mathrm{mM})$. Une respiration non couplée à la phosphorylation existe dans des conditions physiologiques. Elle est maximale après exposition au CCCP, un ionophore à $\mathrm{H}^{+}$, et toujours dépendante de la présence d'une source d'équivalents réducteurs (substrats). Les différents effecteurs sont définis dans la légende du Tableau II.

la mitochondrie. Celle-ci est due à un gradient transmembranaire de protons, maintenu par l'activité de la chaîne respiratoire. La synthèse d'ATP dissipe une partie du gradient et lève l'inhibition de la respiration mitochondriale. Les substrats peuvent alors transmettre des électrons à des accepteurs temporaires (comme le NADH), qui eux-mêmes les transmettent à une chaîne d'accepteurs membranaires, initiant ainsi une série de transferts jusqu'à l'oxygène, leur accepteur final, d'où le nom de " chaîne respiratoire ". Cette cascade énergétique est associée à l'expulsion de protons de la matrice mitochondriale, rétablissant le gradient de protons utilisé pour la synthèse d'ATP. Lorsque ces deux phénomènes sont strictement proportionnels, on dit qu'ils sont couplés. Ainsi la synthèse (et donc la consommation) d'ATP implique à la fois une consommation de substrats et une consommation d'oxygène.

L'encart (p. 272) résume les principes de la phosphorylation oxydative mitochondriale.

Respiration tubulaire. Le Tableau II présente la respiration des tubules en suspension. L'inhibition spécifique de $m / s n^{\circ} 3$ vol. 6 , mars 90
Tableau II

\begin{tabular}{|c|c|c|c|}
\hline \multicolumn{4}{|c|}{$\begin{array}{c}\text { CONTENU ET SYNTHĖSE EN ATP DE SEGMENTS PURIFIÉS } \\
\text { DE NÉPHRONS DE CHIEN }\end{array}$} \\
\hline & $\begin{array}{l}\text { Tubules } \\
\text { proximaux }\end{array}$ & $\begin{array}{l}\text { Anses } \\
\text { larges }\end{array}$ & $\begin{array}{l}\text { Tubules } \\
\text { collecteurs }\end{array}$ \\
\hline $\begin{array}{l}\text { Contenu en ATP } \\
(\mu \mathrm{mol} / \mathrm{g}) \\
\text { Respiration } \\
\left(\mu \mathrm{mol} . \mathrm{g}^{-1} \cdot \mathrm{h}^{-1)}\right.\end{array}$ & $2,0 \pm 0,004(70)$ & $3,3 \pm 0,14(17)$ & $2,6 \pm 0,11(15)$ \\
\hline $\begin{array}{l}\text { Témoin } \\
+ \text { OUA } \\
+ \text { OUA + DCCD } \\
+ \text { OLIGO } \\
+ \text { NYS } \\
+ \text { CCCP } \\
+ \text { roténone }\end{array}$ & $\begin{array}{c}356 \pm 6 \quad(85) \\
228 \pm 6 \quad(67) \\
144 \pm 14(5) \\
137 \pm 6 \quad(6) \\
560 \pm 19(51) \\
895 \pm 45(25) \\
0\end{array}$ & $\begin{array}{rll}342 & \pm 9 & (47) \\
140 & \pm 6 & (39) \\
140 & \pm 6 & (6) \\
140 & \pm 6 & (6) \\
960 & \pm 53(25) \\
1313 & \pm 89(8) \\
& \end{array}$ & $\begin{aligned} & 90 \pm 6 \\
& 31 \pm 7 \\
& 10 \pm 5(8) \\
& 10 \pm 5(5) \\
& 152 \pm 10(7) \\
& 150 \pm 18(5) \\
& 0\end{aligned}$ \\
\hline \multicolumn{4}{|l|}{$\begin{array}{l}\text { Synthèse d'ATP } \\
\left(\mu \mathrm{mol} \cdot \mathrm{g}^{-1} \cdot \mathrm{h}^{-1}\right)\end{array}$} \\
\hline Totale & 1316 & 1212 & 480 \\
\hline $\begin{array}{l}\mathrm{Na}^{+}, \mathrm{K}+\text {-ATPase } \\
\mathrm{H}^{+} \text {-ATPase }\end{array}$ & $\begin{array}{l}768(58 \%) \\
504(38 \%)\end{array}$ & $\begin{aligned} 1212 & (100 \%) \\
& 0(0 \%)\end{aligned}$ & $\begin{array}{ll}354 & (74 \%) \\
126 & (26 \%)\end{array}$ \\
\hline
\end{tabular}

La respiration est mesurée par polarographie dans une chambre oxymétrique en présence de lactate $10 \mathrm{mM}$ (+ glutamine $10 \mathrm{mM}$ pour les tubules proximaux). OUA = ouabaine $1 \mathrm{mM} ; D C C D=1,3$-dicyclohexylcarbodiimide $25 \mu \mathrm{M}$; Oligo = oligomycine 2,5 $\times 10^{-7} \mathrm{M} ; \quad N Y S=$ nystatine $0,5 \mathrm{mM} ; C C C P=$ carbonylcyanide $\mathrm{m}$. chlorophenylhydrazone $0,1 \mathrm{mM}$. La synthèse d'ATP est dérivée de la respiration phosphorylante (respiration totale - OLIGO) $\times 6$. Les changements de respiration phosphorylante produits par OUA et OUA + DCCD ont servi, respectivement, à estimer la synthèse d'ATP requise pour l'activité de la $\mathrm{Na}^{+}, \mathrm{K}^{+}$-ATPase et la $\mathrm{H}^{+}$-ATPase membranaire in situ. La roténone est un inhibiteur de la chaîne respiratoire. Les données de respiration et de synthèse d'ATP sont exprimées en micromoles par gramme de tissu frais et par heure. Moyennes \pm ESM (n). 


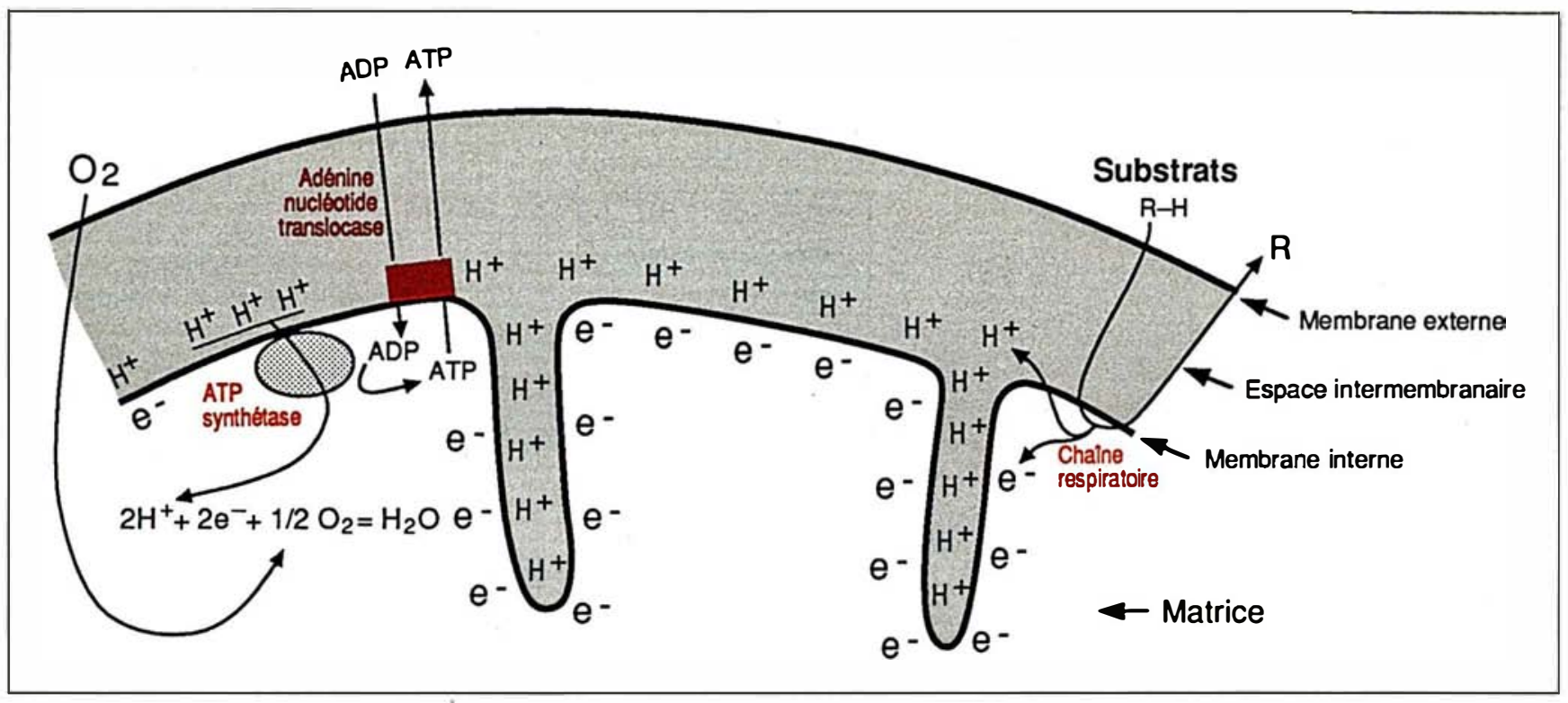

Principe de la phosphorylation oxydative mitochondriale. Les substrats réduits RH cèdent leurs électrons à une série d'accepteurs en cascade, chacune des réactions produisant de l'énergie utilisée pour expulser les protons $\mathrm{H}^{+}$à l'extérieur de la membrane interne alors que les électrons $e^{-}$demeurent dans la matrice. Le dernier accepteur est l'oxygène $\mathrm{O}_{2}$, réduit en $\mathrm{O}_{2}^{-}$. Le gradient de proton créé est à l'origine d'une différence de potentiel entre les deux faces de la membrane interne. Le retour de protons dans la matrice, mû par le gradient, crée de l'énergie utilisée par l'ATP synthétase pour phosphoryler l'ADP en ATP (force protonomotrice); ces deux nucléotides sont échangés par l'intermédiaire d'une adénine nucléotide translocase. L'ion $\mathrm{H}^{+}$ayant pénétré dans la matrice se combine à l'ion $\mathrm{O}_{\overline{2}}$ pour donner de l'eau $\mathrm{H}_{2} \mathrm{O}$. L'ATP produit est utilisé, notamment, via les pompes ioniques (ATPase $\mathrm{Na}^{+}-\mathrm{K}^{+}$, ATPase $\mathrm{H}^{+}$, entre autres). Toute consommation $d^{\prime}$ ATP $\mathrm{s}^{\prime}$ accompagne d'une augmentation compensatrice de la respiration. La destruction du gradient de protons inhibe la synthèse d'ATP et déréprime la respiration, qui est donc "découplée ". Le blocage de l'entrée des protons au niveau du site "phosphorylant " (inhibition de l'ATP synthétase par l'oligomycine) inhibe la respiration.

l'ATP-synthétase par l'oligomycine ne supprime pas toute la respiration, démontrant qu'une entrée de protons survient par d'autres sites que par le site phosphorylant : $38 \%$ de la respiration spontanée sont dus à cette activité respiratoire physiologiquement " découplée " dans les segments tubulaires riches en mitochondries, le tubule proximal et l'anse large. Nous appellerons " respiration non phosphorylante " cette respiration non inhibée par l'oligomycine (38\%) et "respiration phosphorylante " la respiration abolie par l'inhibition de la synthèse d'ATP (62 \%) (figure 2). La totalité des activités ATPasiques des cellules se trouve donc exprimée dans cette dernière fraction de la respiration cellulaire. L'addition d'ouabaïne supprime $60 \%$ de la synthèse d'ATP dans les tubules proximaux, indiquant que le transport sodé réalisé par la $\mathrm{Na}^{+}, \mathrm{K}^{+}$-ATPase $\mathrm{y}$ représente plus de la moitié du tra- vail cellulaire; l'autre fraction de $40 \%$ est sensible à l'addition ultérieure de faibles concentrations de DCCD, un inhibiteur puissant (mais non spécifique) de la $\mathrm{H}^{+}$-ATPase, indiquant que la sécrétion de protons pourrait consommer une fraction importante de la synthèse d'ATP dans le tube proximal et dans le tubule collecteur. Au contraire, le transport sodé consomme virtuellement tout l'ATP utilisé dans les anses larges (Tableau II) où on ne retrouve pas de respiration sensible au DCCD. Ces chiffres sont en accord avec les mesures des ATPases de transport présentées plus haut (Tableau I).

Une stimulation de la respiration liée à l'activité ATPasique de la $\mathrm{Na}^{+}$, $\mathrm{K}^{+}$-ATPase est produite par l'ionophore à cations $\left(\mathrm{Na}^{+}, \mathrm{K}^{+}\right)$nystatine (Tableau II et figure 2), démontrant que la synthèse d'ADP peut s'adapter rapidement à une production accrue d'ADP et de Pi. La respiration complètement découplée par l'ionophore à protons CCCP excède la respiration couplée maximale (Tableau II et figure 2), indiquant que la disponibilité en oxygène et en substrats ne limite normalement pas la synthèse d'ATP dans ces tubules. Enfin la figure 2 montre que l'apport de substrats module la respiration cellulaire. Les raisons de cette observation sont exposées plus loin.

\section{Particularités des tubules en suspension}

Gardant en tête ce schéma des relations entre transport et respiration, examinons les particularités métaboliques imposées par la technique de suspension tubulaire.

Un travail non limité par la filtration glomérulaire. Le travail de réabsorption tubulaire proximal est normalement limité par le taux de fil- 
tration glomérulaire, qui ne permet qu'à $20 \%$ de l'ultrafiltrat plasmatique de se retrouver dans la lumière tubulaire et d'y être en grande partie réabsorbée (figure 3, panneau de gauche). In situ, cela limite donc l'activité de la $\mathrm{Na}^{+}, \mathrm{K}^{+}$-ATPase, la synthèse d'ATP et la respiration proximale. Un raisonnement analogue peut s'appliquer à la sécrétion de protons et à la réabsorption des bicarbonates liées à l'activité de la $\mathrm{H}^{+}$-ATPase. De même, l'anse large et le tube collecteur sont normalement limités dans leur travail par la disponibilité en sodium, en potassium et en chlore dans la lumière tubulaire (figure 3, panneau de gauche).

$\mathrm{Au}$ contraire, les segments tubulaires en suspension sont privés de la barrière glomérulaire (figure 3 , panneau de droite). Ils transporteront donc de façon illimitée les éléments réabsorbables qui se présentent dans leur lumière. Le taux d'utilisation d'ATP par les ATPases de transport, et partant la synthèse d'ATP par millimètre de tubule, seront donc plus grands dans les suspensions qu'in situ. Chacun des segments (figure 3, panneau de droite) sera métaboliquement plus actif qu'in situ, et consommera par conséquent plus d'oxygène et de substrats que dans l'organe.

Une accumulation intracellulaire anormale de substrats. Dans les conditions physiologiques, les substances organiques réabsorbées par les bordures en brosse le sont dans les premiers millimètres du tube proximal. Le transport est non saturé dans ces conditions et la concentration de substrat diminue rapidement dans la lumière, limitant rapidement le transport. Au contraìre, dans les suspensions tubulaires, les transporteurs localisés sur les bordures en brosse sont exposés à une concentration uniforme et à une quantité infinie de substrats. Ceci implique quc les transporteurs pourront fonctionner à vitesse maximale ( $\mathrm{V} \max$ ) de façon continue. L'apport de substrats dans la cellule excédera donc celui de la situation physiologique, ayant pour conséquence une accumulation intracellulaire de substrats, même lorsque les tubules sont incubés dans un milieu contenant une concentration de substrat mimant celle du plasma. Ceci est illustré sur la figure 4 (p. 274)

$\mathrm{m} / \mathrm{s} n^{\circ} 3$ vol. 6 , mars 90 qui décrit les changements de concentration de glutamine et de glutamate dans le milieu d'incubation et dans les cellules de tubules proximaux de rats en fonction du temps. On peut voir qu'en dépit de l'utilisation d'une concentration "physiologique " de glutamine (0,5-1 mM), la glutamine cellulaire s'élève rapidement à $4 \mathrm{mM}$ soit environ deux fois la concentration physiologique [10, 11]. Cela aura deux conséquences. La première est que toutes les voies métaboliques réglées par la concen- tration intracellulaire de ces substrats seront recrutées d'une façon anormale. Si ces voies métaboliques conduisent à une synthèse d'ATP, le mélange de substrats supportant la synthèse d'ATP sera modifié in vitro $[1,12]$. L'étude du métabolisme d'un acide aminé comme la glutamine avec ce système correspond donc à l'étude in vivo d'un animal chargé en glutamine [12]. Cette considération est importante puisque des voies métaboliques non recrutées physiologiquement pourront apparaî-

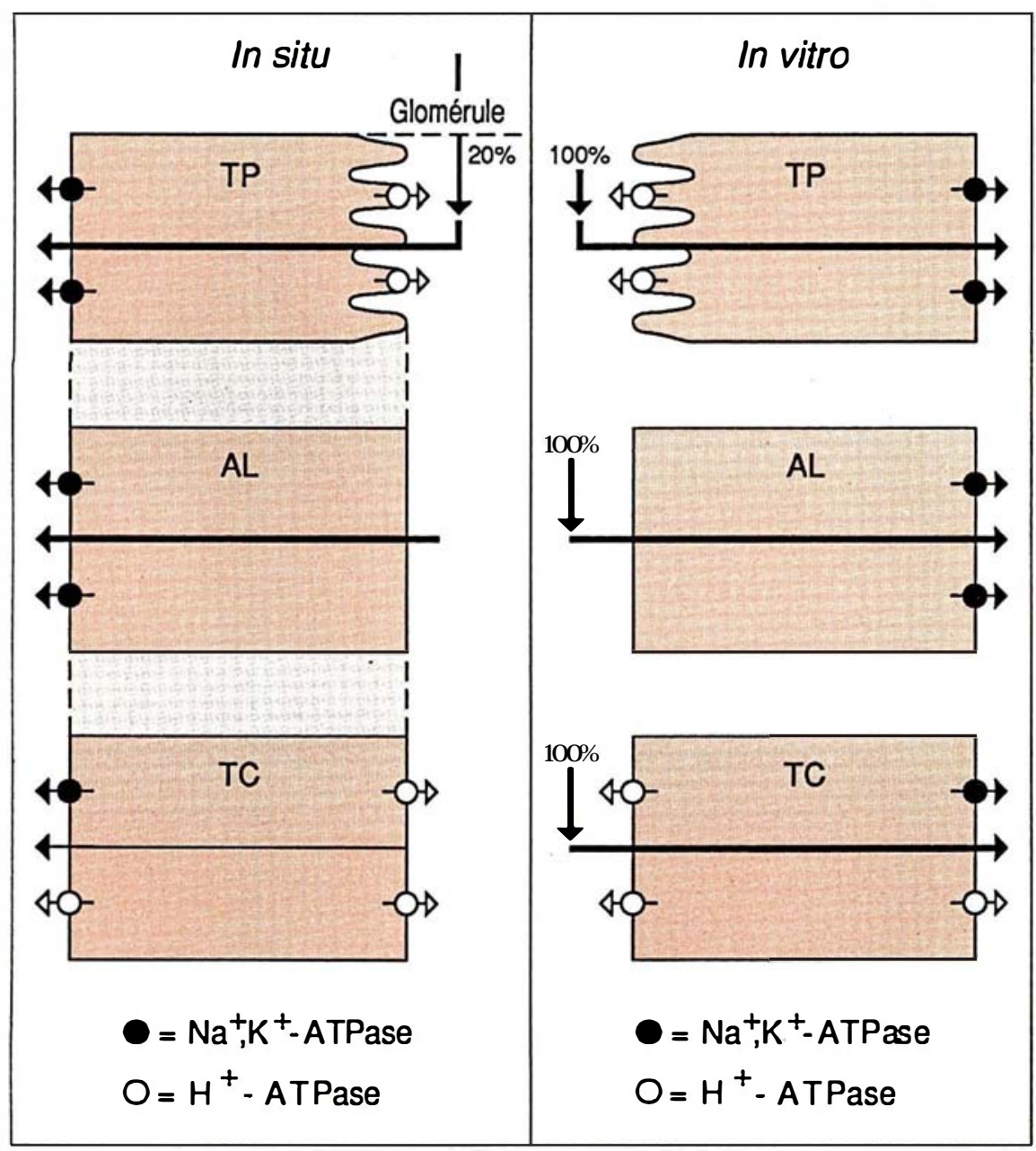

Figure 3. Différences entre les conditions de transport par les segments de néphron in situ et in vitro. Le panneau de gauche présente le tube proximal (TP), l'anse large ( $A L$ ) et le tube collecteur papillaire (TC) dans la continuité $d^{\prime} u n$ seul néphron (pointillé vertical) dont le filtre glomérulaire est indiqué (pointillé horizontal). La filtration glomérulaire et la réabsorption limitent le transport des segments distaux. Le panneau de droite présente la condition obtenue avec des segments isolés de néphrons où ces limites ne jouent plus. Les petites flèches noires indiquent la direction du transport sodé et les flèches blanches, celui du transport de protons. 


\section{RÉFÉRENCES}

9. Manillier C, Vinay P, Lalonde L, Gougoux A. ATP turnover and renal response of dog tubules to $\mathrm{pH}$ changes in vitro. $A m$ $J$ Physiol 1986 ; 251 : 919-32.

10. Vinay $\mathrm{P}$, René de Cotret $\mathrm{P}$, Schwartz $\mathrm{H}$, Gougoux A, Lemieux G. Cell transport and metabolism of glutamine by proximal and/or distal tubules of rat kidney. In : Morel F, ed. Biochemistry of kidney functions. Symposium Inserm $\mathrm{n}^{\circ} 21$. Amsterdam : Elsevier, $1982 ; 167-76$.

11. Vinay $\mathrm{P}$, Allignet E, Pichette $\mathrm{C}$, Watford M, Lemieux G, Gougoux A. Changes in renal metabolic profile and ammoniagenesis during acute and chronic metabolic acidosis in dog and rat. Kidney Int 1980 ; $17: 312-25$

12. Gougoux A, Vinay $\mathrm{P}$, Halperin ML. Regulation of renal ammoniagenesis in the dog with chronic metabolic acidosis : effect of a glutamine load. Am J Physiol 1985 ; 249 : 745-52.

13. Cornell NW, Lund P. Krebs HA. The effect of lysine on gluconeogenesis from lactate in rat hepatocytes. Biochem J 1974; 142 : 327-37.

14. Vinay P, Sénécal J, Noël J, Tejedor $A$, Berteloot A, Gougoux A. Basolateral transport of lactate in dog thick ascending limbs. Am J Physiol 1990 (sous presse).

15. Noël J, Tejedor A, Vinay P, Laprade $\mathrm{R}$, Gougoux A. BBM $\mathrm{H}^{+}$ATPase activity in the dog kidney : modulation by substrate availability. Kidney Int 1990 ; 37 (sous

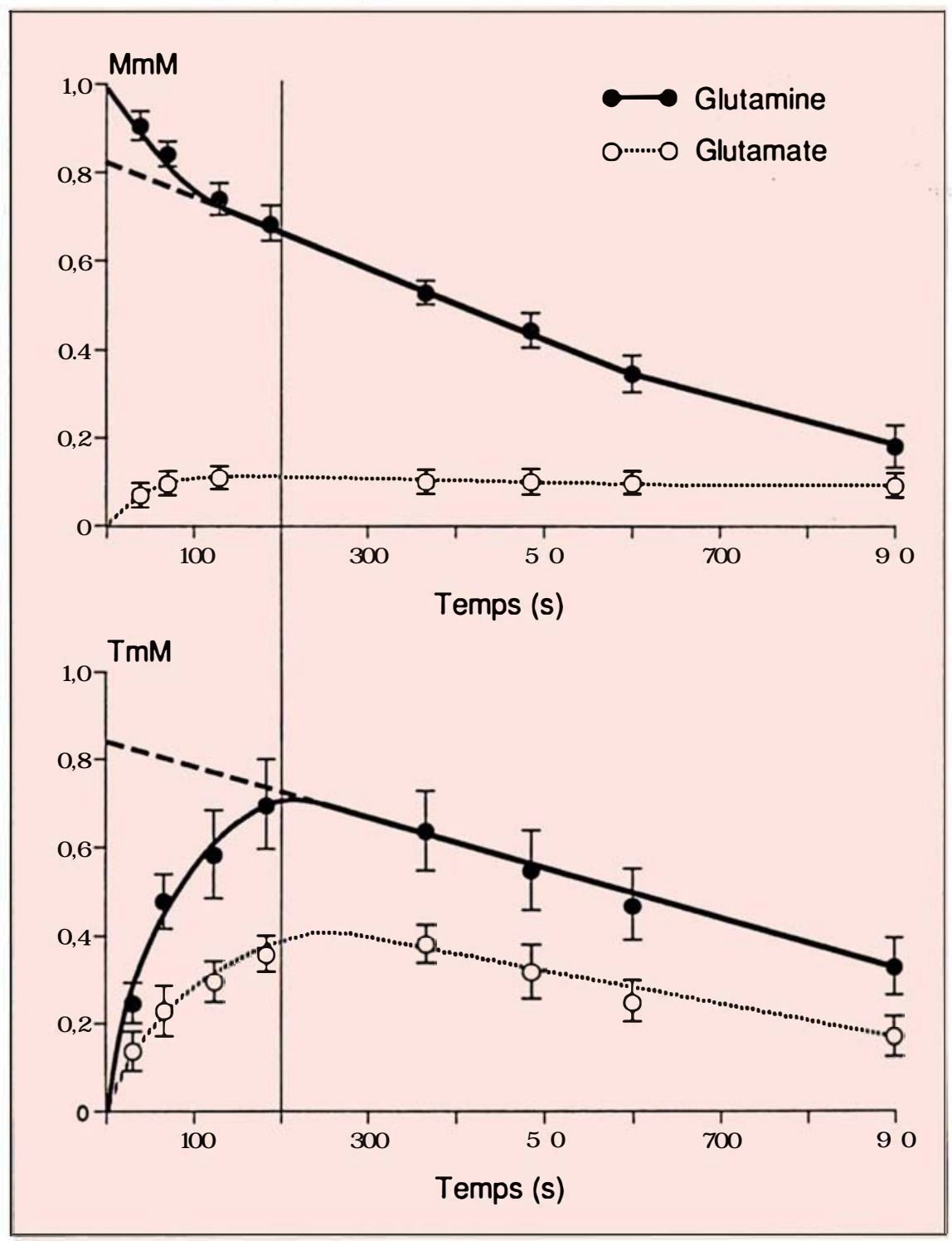

Figure 4. Évolution des concentrations de glutamine et de glutamate (exprimées en $\mathrm{mM}$ ) dans le milieu d'incubation (M) et dans le milieu tissulaire (T) de tubes proximaux de reins de rat incubés en présence de glutamine $1 \mathrm{mM}$. Une accumulation tissulaire de glutamine et de glutamate est observée durant les 200 premières secondes (panneau du bas), altérant la dynamique d'extraction de la glutamine du milieu (panneau du haut). Notez les concentrations intracellulaires supraphysiologiques de glutamate produites en dépit des concentrations extracellulaires physiologiques.

tre in vitro. Un exemple de ceci est donné sur la figure 4. Lorsque la glutamine est présentée à la cellule, une concentration importante de glutamine est créée dans les cellules proximales. Cela donne lieu à une production intracellulaire de glutamate. Comme il n'y a pas de glutamate à l'extérieur de la cellule, c'est-à-dire dans le milieu d'incubation, un gra- dient permanent existe pour cet acide aminé entre la cellule et son environnement. Un efflux de glutamate s'ensuit, et le métabolisme initial de la glutamine sera surtout utilisé pour établir une concentration critique de glutamate dans le milieu d'incubation. Ce phénomène cesse lorsque les transporteurs de glutamate établissent un nouvel équilibre entre le gluta- 
Tableau III

EFFET DE LA PRÉPARATION ET DE L'INCUBATION DE TUBULES ISOLÉS SUR LE CONTENU TISSULAIRE EN ACIDES AMINÉS

\begin{tabular}{|c|c|c|c|c|}
\hline Acides aminés & $\begin{array}{l}\text { Reins obtenus par } \\
\text { congélation instantanée } \\
\qquad(n=5)\end{array}$ & $\begin{array}{l}\text { Tubules isolés } \\
\text { fraîchement préparés } \\
\qquad(n=4)\end{array}$ & $\begin{array}{c}\text { Tubules isolés } \\
\text { incubation de } 30 \text { minutes } \\
\text { sans substrat } \\
(n=4)\end{array}$ & $\begin{array}{l}\text { Tubules isolés } \\
\text { incubation de } 30 \text { minutes } \\
\text { avec lactate } 10 \mathrm{mM} \\
(\mathrm{n}=4)\end{array}$ \\
\hline Leucine & $0,13 \pm 0,0$ & $2,40 \pm 0,31$ & $4,11 \pm 0,48$ & $4,57 \pm 0,38$ \\
\hline Isoleucine & $0,07 \pm 0,0$ & $1,47 \pm 0,20$ & $2,39 \pm 0,34$ & $2,71 \pm 0,17$ \\
\hline Valine & $0,12 \pm 0,01$ & $2,33 \pm 0,26$ & $4,02 \pm 0,32$ & $4,41 \pm 0,36$ \\
\hline Sérine & $0,88 \pm 0,21$ & $3,07 \pm 0,18$ & $8,65 \pm 0,46$ & $8,69 \pm 0,61$ \\
\hline Histidine & $0,08 \pm 0,08$ & $0,78 \pm 0,08$ & $1,65 \pm 0,13$ & $1,68 \pm 0,13$ \\
\hline Thréonine & $0,62 \pm 0,11$ & $1,61 \pm 0,09$ & $3,61 \pm 0,21$ & $3,67 \pm 0,29$ \\
\hline Tyrosine & $0,04 \pm 0,002$ & $1,02 \pm 0,12$ & $2,03 \pm 0,12$ & $2,04 \pm 0,19$ \\
\hline Phénylalanine & $0,02 \pm 0,01$ & $1,13 \pm 0,17$ & $2,19 \pm 0,12$ & $2,19 \pm 0,19$ \\
\hline Méthionine & $0,13 \pm 0,01$ & $1,33 \pm 0,17$ & $2,26 \pm 0,22$ & $2,44 \pm 0,43$ \\
\hline Glycine & $1,13 \pm 0,33$ & $2,17 \pm 0,08$ & $3,86 \pm 0,42$ & $3,93 \pm 0,48$ \\
\hline Alanine & $0,34 \pm 0,03$ & $2,47 \pm 0,14$ & $3,55 \pm 0,42$ & $5,81 \pm 0,46$ \\
\hline Phosphoéthanolamine & $2,49 \pm 0,06$ & $1,81 \pm 0,28$ & $2,27 \pm 0,40$ & $1,95 \pm 0,38$ \\
\hline Lysine & $0,21 \pm 0,80$ & $3,16 \pm 0,43$ & $3,68 \pm 0,18$ & $3,90 \pm 0,49$ \\
\hline Taurine & $0,28 \pm 0,01$ & $1,04 \pm 0,15$ & $0,78 \pm 0,16$ & $0,55 \pm 0,11$ \\
\hline Ornithine & $0,02 \pm 0,004$ & $0,19 \pm 0,02$ & $0,11 \pm 0,01$ & $0,09 \pm 0,02$ \\
\hline Aspartate & $1,68 \pm 0,39$ & $7,68 \pm 0,81$ & $2,46 \pm 0,19$ & $3,11 \pm 0,28$ \\
\hline Arginine & $0,11 \pm 0,01$ & $2,71 \pm 0,18$ & $2,06 \pm 0,18$ & $2,28 \pm 0,21$ \\
\hline Glutamate & $4,91 \pm 0,19$ & $7,74 \pm 0,61$ & $4,22 \pm 0,64$ & $9,61 \pm 0,71$ \\
\hline Glutamine & $1,15 . \pm 0,03$ & $0,62 \pm 0,09$ & $0,57 \pm 0,09$ & $2,14 \pm 0,19$ \\
\hline Proline & $0,05 \pm 0,02$ & $1,45 \pm 0,18$ & $1,00 \pm 0,20$ & $2,85 \pm 0,48$ \\
\hline
\end{tabular}

Le contenu en acides aminés de flacons d'incubation de fragments tubulaires proximaux de rat incubés en présence ou en absence de substrats, et rapporté en micromoles par gramme de tissus, a été comparé au contenu de fragments tubulaires fraîchement séparés et à celui de reins de rats prélevés par congélation instantanée. Moyenne \pm ESM. Les mesures ont été obtenues au moyen $d^{\prime} u n$ autoanalyseur d'acides aminés.

mate intra- et extracellulaire (figure 4). Ainsi, le métabolisme de la glutamine vers la formation de glutamate pourra varier selon la quantité de glutamine présentée et selon le volume d'incubation. Si l'on ne tient pas compte de ce phénomène, on tirera des conclusions erronées sur la contribution respective des différentes voies métaboliques qui participent au métabolisme de la glutamine.

Des tubules initialement chargés en acides aminés. Au cours de la préparation de la suspension tubulaire, le tissu est exposé à l'action de collagénase et de protéases. Sans cellesci, l'activité collagénase ne suffit pas à réaliser la digestion tissulaire. Cette activité hydrolytique s'exerce sur les protéines libérées des cellules détruites au cours du processus de préparation des tranches tissulaires. Il s'ensuit une protéolyse qui élève la concentration d'acides aminés dans le milieu de digestion. Étant donné la $m / s n^{\circ} 3$ vol. 6 , mars 90 présence des mécanismes puissants de transport membranaire exposés à ce milieu, la concentration d'acides aminés s'accroît rapidement dans le milieu intracellulaire proximal (Tableau III). Ainsi des tubules fraîchement préparés seront enrichis en acides aminés, et cela pourra avoir un impact sur le métabolisme des composés organiques étudiés avec cette préparation. Au contraire, la préparation d'hépatocytes isolés est initialement appauvrie en acides aminés libres du fait de leur dialyse dans le milieu de digestion (et de lavage) en absence de ces transporteurs membranaires spécialisés [13]. Cette différence explique la stimulation spécifique de la lysine sur la gluconéogenèse retrouvée dans les hépatocytes [13], mais non dans les tubules proximaux.

Effet de l'apport de substrats sur la pompe à sodium. Au niveau des tubules proximaux, les mécanismes de réabsorption tubulaire mettent en cause un co-transport sodiumsubstrat. Ainsi l'élévation progressive de glutamine, de lactate ou de glucose dans le milieu d'incubation de tubules proximaux en suspension va élever progressivement la respiration cellulaire (figure 5, p. 276). Cette stimulation traduit en partie le travail accru de la $\mathrm{Na}^{+}, \mathrm{K}^{+}$-ATPase secondaire aux phénomènes de cotransport et est supprimée par l'inhibition de ceux-ci. Le fait d'ajouter des substrats exogènes aux tubules modifie donc le travail effectué par la cellule, et ses besoins énergétiques. Cet effet sera modulé par le nombre de transporteurs présents dans la membrane, par leurs caractéristiques cinétiques et par la concentration de substrats imposée.

Les anses larges, quant à elles, ne possèdent pas de bordure en brosse et le transport de leur substrat naturel, le lactate, n'y implique pas un co-transport sodé. Pourtant l'addition de lactate, mais non pas celui de glu- 


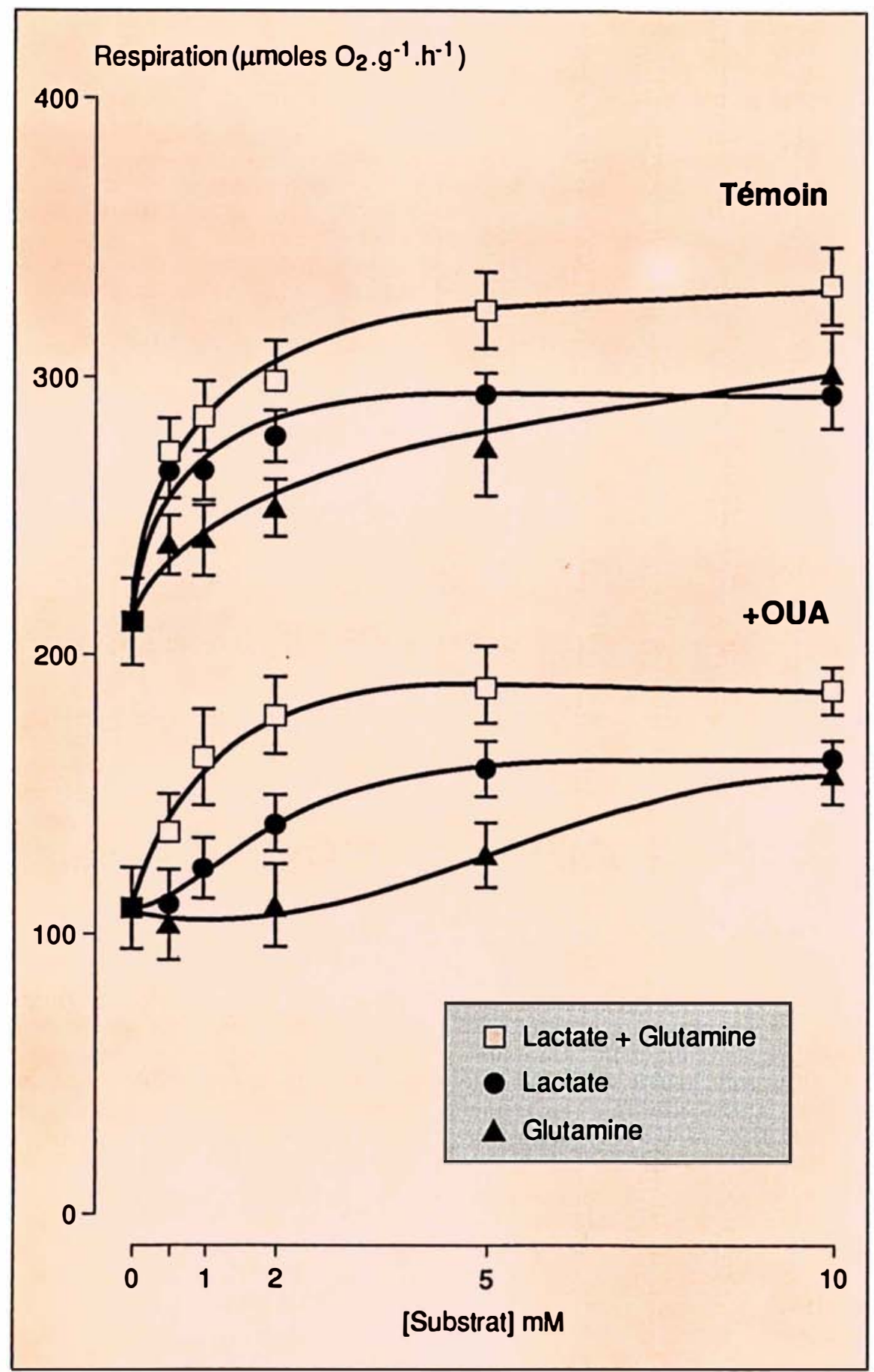

Figure 5. Accroissement et saturation de la respiration totale et de la respiration observée en présence d'ouabaïne (OUA, $1 \mathrm{mM}$ ), provoqués par I'addition de substrats dans les tubules corticaux de chien. La respiration sensible à l'ouabaine est obtenue par différence des points expérimentaux des deux courbes. La respiration est mesurée par polarographie dans une cose, y détermine une stimulation de l'activité $\mathrm{Na}^{+}, \mathrm{K}^{+}$-ATPase (figure 6). Nous avons montré que ceci était dû à une activation indirecte de la pompe à sodium. En effet le lactate pénètre dans la cellule au moyen d'un échange lactate-bicarbonate [14], et le déséquilibre en bicarbonate créé favorise à son tour un échange bicarbonate-chlore. L'effet net est donc de faciliter la sortie de chlore et de dissiper le gradient de chlore créé par le transport épithélial dans cette cellule. Cela a comme effet de faciliter l'entrée cellulaire de $\mathrm{NaCl}$ par le transporteur luminal et donc d'activer le travail de la pompe. Ici encore, le fait d'ajouter des substrats exogènes aux tubules modifie le travail effectué par la cellule, et donc ses besoins énergétiques.

Effet de l'apport de substrat sur le transport de $\mathrm{H}^{+}$. Le transport en grande quantité de substrats cotransportés avec du sodium pourra causer une dépolarisation cellulaire et ainsi influencer la concentration intracellulaire de bicarbonate. Ceci aura un effet sur le $\mathrm{pH}$ intracellulaire du tubule proximal. Le transport de glucose, par exemple, alcalinise la cellule proximale (figure $7, p .278$ ). Ceci interfere avec la fonction des mécanismes réglant le $\mathrm{pH}$ intracellulaire et plus spécifiquement avec l'activité de la $\mathrm{H}^{+}$-ATPase membranaire, représentée par la respiration sensible au DCCD [15]. Il en résulte une baisse de la synthèse d'ATP (et de respiration) reliée à cette fonction. $\mathrm{Au}$ contraire, l'addition de lactate acidifie la cellule, stimule l'activité de la $\mathrm{H}^{+}$-ATPase, et la respiration sensible au DCCD. L'addition de glutamine ne change pas cette fonction (figure 7).

Effet découplant des substrats. L'entrée des substrats dans la mitochondrie utilise fréquemment le gradient de protons créé de part et d'autre de la membrane mitochondriale. Ainsi, un co-transporteur de pyruvate $-\mathrm{H}^{+}$, sensible au cyanocinnarnate, internalise le pyruvate dans la mitochondrie où se produit son métabolisme en acétyl-CoA puis en $\mathrm{CO}_{2}$. Le transport de pyruvate entraîne donc une entrée de protons dans la mitochondrie qui ne passe pas par l'ATP synthétase : il s'agit d'un effet découplant des substrats. 


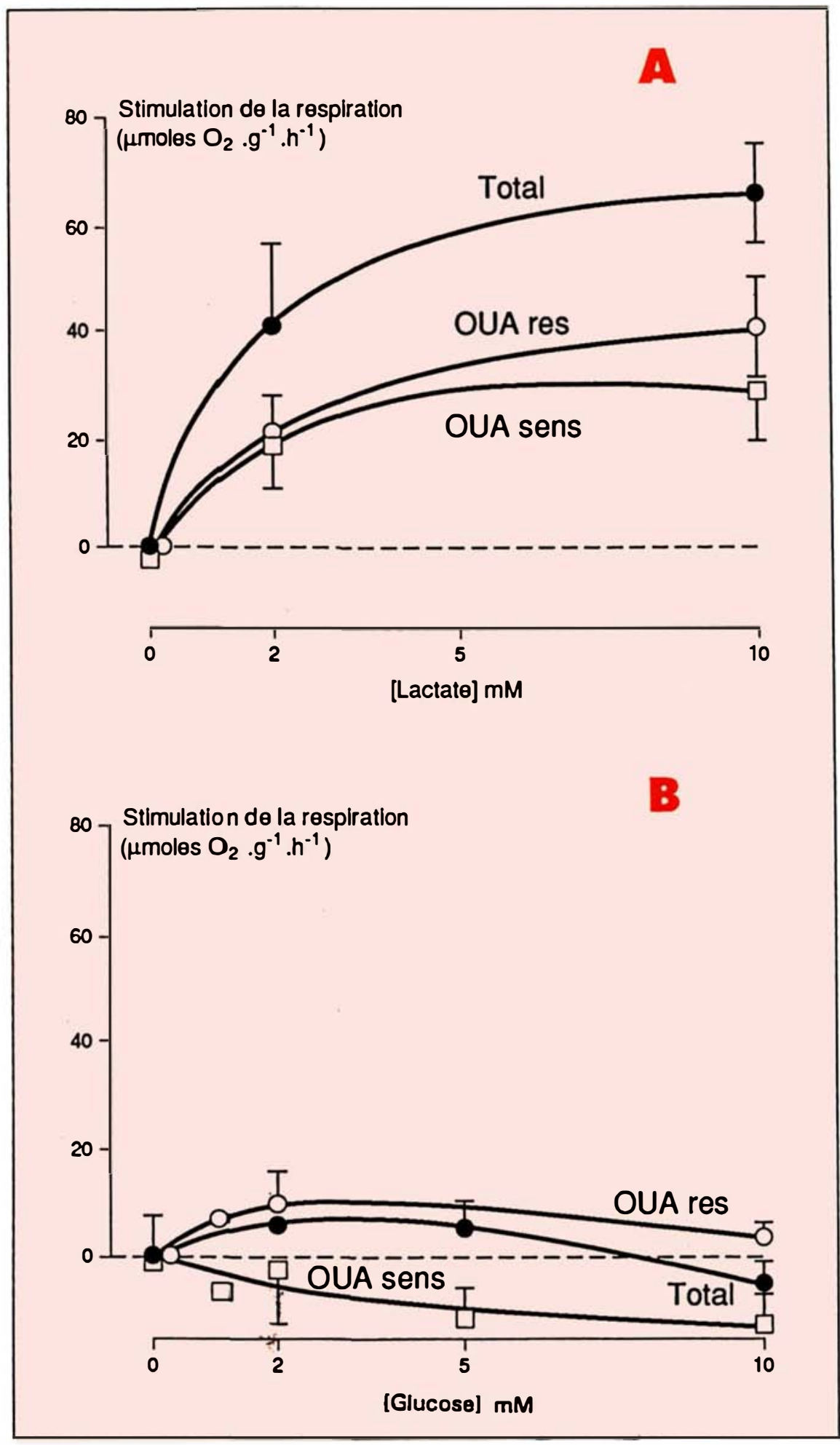

Figure 6. Stimulation de la respiration sensible et résistante à l'ouabaïne (OUA, 1 mM) provoquée par l'addition de lactate (A), mais non de glucose (B), dans les anses larges de chien. Moyennes $\pm E S M(n=3)$. $\mathrm{m} / \mathrm{s} n^{\circ} 3 \mathrm{vol}$. 6 , mars 90
Lorsqu'une entrée cellulaire massive de lactate engendre une concentration importante de pyruvate dans la cellule (de par l'état d'équilibre de la lactate déshydrogénase), celui-ci entre dans la mitochondrie, entraînant une activation découplée de la chaîne respiratoire : la consommation d'oxygène insensible à l'oligomycine s'accroît (figure 8, p. 278). De même, la disponibilité en glutamine et la déamidation mitochondriale de celleci dans le compartiment mitochondrial aura un effet analogue: le $\mathrm{NH}_{4}^{+}$produit, se dissociant en $\mathrm{NH}_{3}$ et $\mathrm{H}^{+}$, stimule la respiration mitochondriale. On devra tenir compte de cet effet non spécifique des substrats sur la respiration au cours de l'analyse de la synthèse d'ATP de ces segments tubulaires.

Le potentiel d'oxydo-réduction (rédox). Certains substrats font partie d'un couple rédox proche de l'équilibre thermodynamique. Ainsi, le lactate et le pyruvate sont normalement présents dans la cellule en proportion d'équilibre de 10 pour 1 . Tout apport de lactate entraîne automatiquement une formation de pyruvate pour maintenir cet équilibre. Lorsque du lactate seul est ajouté à des tubules, une génération rapide de pyruvate se produit avec une bouffée de production de NADH dans le cytoplasme. Ce NADH pourra devenir transitoirement la principale source d'électrons pour la chaîne respiratoire. L'utilisation normale des substrats et le flux dans les voies métaboliques impliquant des couples rédox pourront donc être transitoirement modifiés [5]. Ceci peut être évité en ajoutant du pyruvate au lactate dans les proportions 1:10 dans le milieu d'incubation.

\section{Conclusions}

L'utilisation de tubules en suspension ouvre la voie à une compréhension plus fine des rôles métaboliques variés joués par les différents segments du néphron. Ces préparations ont l'avantage de permettre l'étude des flux métaboliques et de leur régulation. Cependant les renseignements fournis doivent être interprétés en tenant compte des particularités fonctionnelles spécifiques des suspensions tubulaires. L'approche idéale utilisera différents niveaux d'organisation bio- 


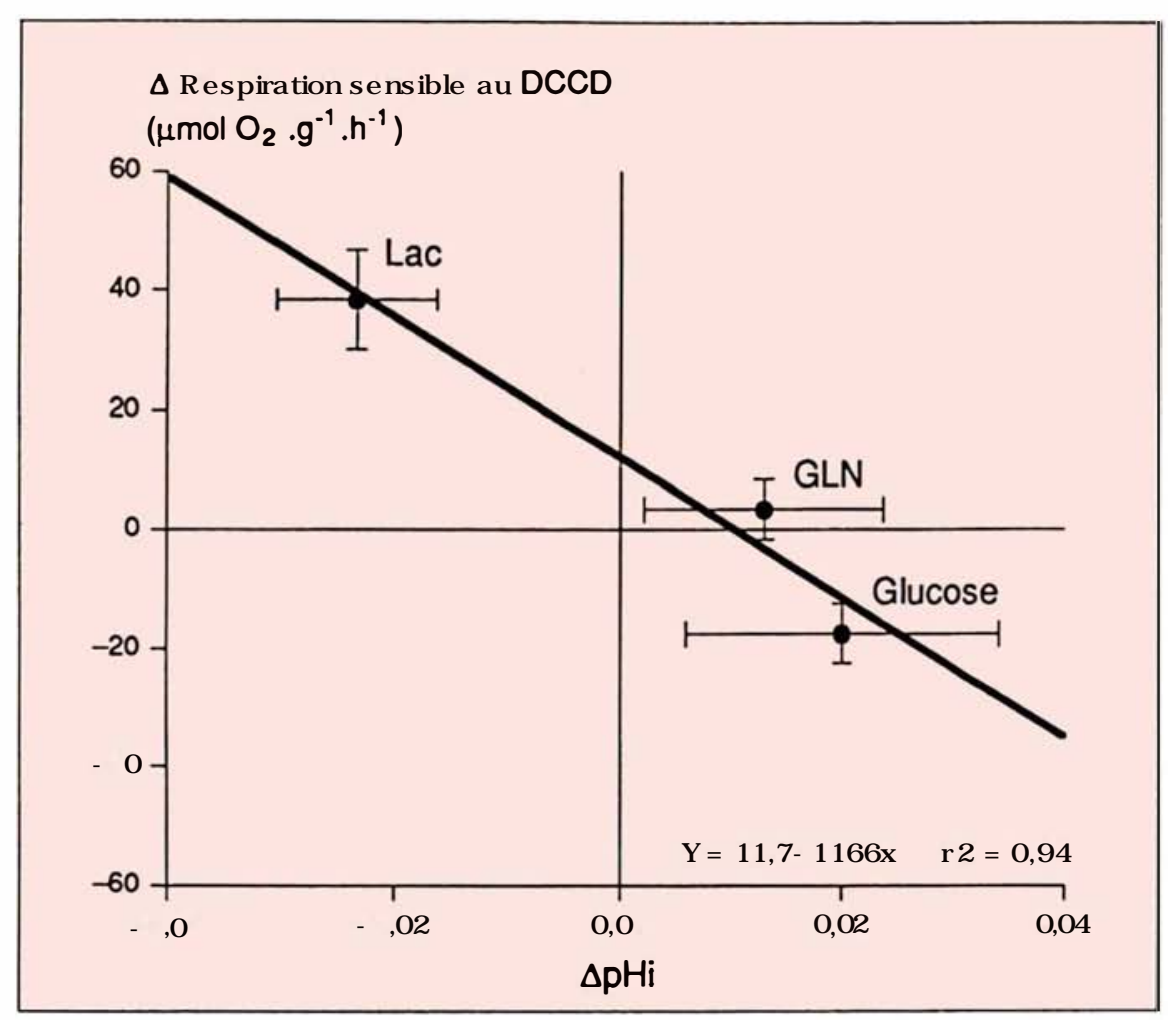

Figure 7. Corrélation entre la respiration sensible au DCCD, reflétant l'activité de la $H^{+-A}$-A Pase, et le changement de pH intracellulaire ( $\left.\Delta \mathrm{pHi}\right)$ induit par l'addition de substrats $(10 \mathrm{mM})$ à des tubules proximaux de chien. Le pHi est mesuré par spectrofluorométrie en utilisant le BCECF, une sonde sensible au $\mathrm{pH}$. Moyennes $\pm E S M(n=6)$.

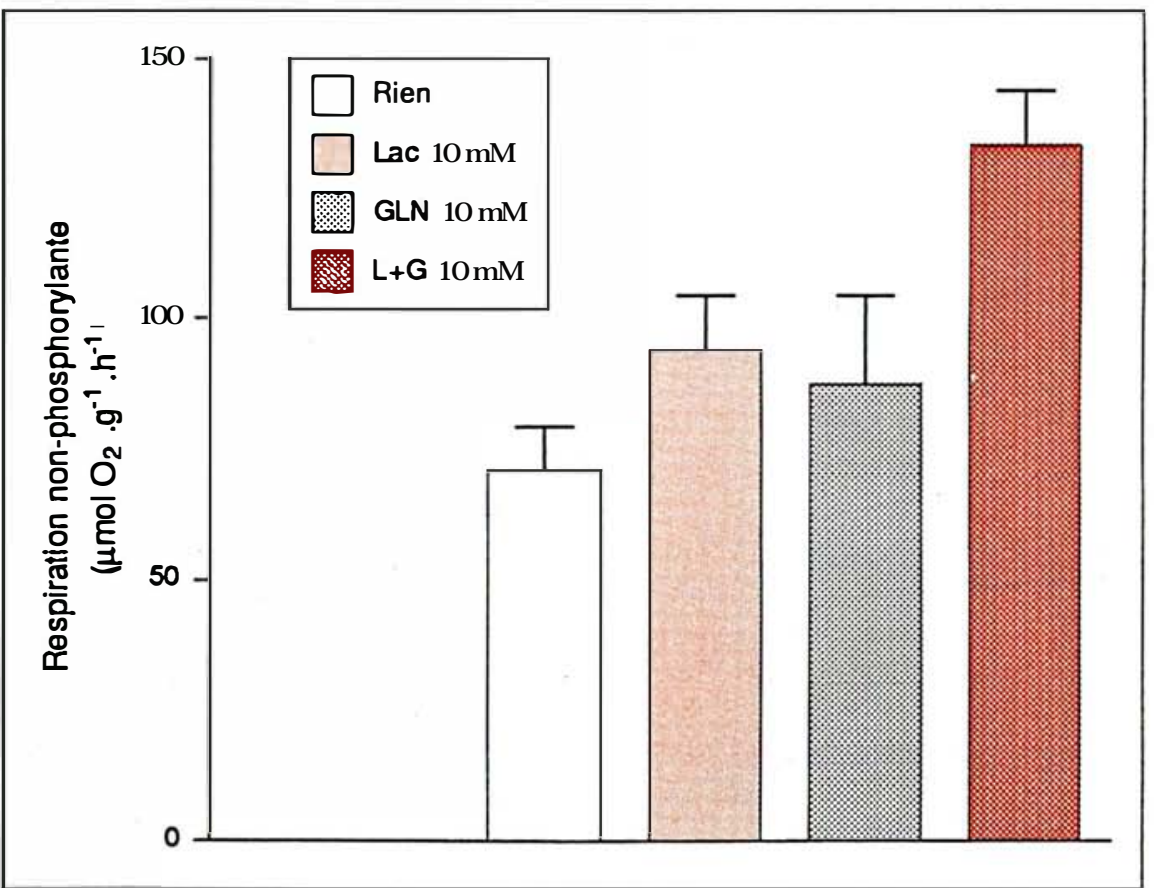

Figure 8. Respiration non phosphorylante observée avant (rien) et après addition de lactate (Lac), de glutamine (GLN) ou d'un mélange (L + G) à des tubules proximaux de chien. La respiration est mesurée en présence d'oligomycine $2,5 \times 10^{-7} \mathrm{M}$. Moyennes \pm ESM $(n=6)$. logique (in vivo, organes perfusés, tubules en suspension ou microdisséqués, cellules en culture, homogénats, organelles cellulaires, vésicules membranaires). Ceci permettra d'intégrer les observations obtenues à chaque niveau dans un contexte physiologique et d'échapper aux biais imposés par ces techniques d'étude elles-mêmes

\section{Summary}

In vitro studies of renal cell metabolism : advantages and pitfalls

The use of suspensions of freshly isolated tubular segments originating from different parts of the nephron (proximal tubules, thick ascending limbs, collecting ducts) allows the investigation of the multiple and heterogeneous metabolic functions of this organ. However the in vitro technique modifies the functional and biochemical situation of the renal cells. An analysis of some of these specific changes is presented. The metabolic consequences of the loss of the glomerular barrier, leading to an increment in the work performed by the cells and to a supraphysiological accumulation of transported substrates, leads to a change in ATP turnover and modifies the choice of substrates and pathways used to produce the energy required for transport. Substrateregulated metabolic pathways may be recruited in a fashion normally unseen in vivo. The aminoacid content of freshly prepared tubules is supraphysiological. The addition of metabolic substrates changes directly or indirectly the rate of sodium or proton transport by the cells and may alter the cell $\mathrm{pH}$. A large physiological " uncoupling effect " of certain substrates is also noted. Finally, large redox changes can be imposed by the choice of substrates and incubation conditions. The understanding of these specific characteristics is required to avoid potential pitfalls in the interpretation of metabolic data generated with renal tubule suspensions. 